\title{
Spectral Element Numerical Investigation of Flow between Three Cylinders in an Equilateral-Triangular Arrangement with Different Spacing Distances
}

\author{
Zhenzhong Bao $\mathbb{D}$, Guoliang Qin $(\mathbb{D})$, Wenqiang He, and Yazhou Wang \\ School of Energy and Power Engineering, Xi'an Jiaotong University, Xi'an, Shanxi 710049, China \\ Correspondence should be addressed to Guoliang Qin; glqin@xjtu.edu.cn
}

Received 21 February 2018; Revised 11 April 2018; Accepted 22 April 2018; Published 23 May 2018

Academic Editor: Adam Glowacz

Copyright (C) 2018 Zhenzhong Bao et al. This is an open access article distributed under the Creative Commons Attribution License, which permits unrestricted use, distribution, and reproduction in any medium, provided the original work is properly cited.

Two-dimensional incompressible Navier-Stokes equations are numerically solved using the high resolution spectral element method at Reynolds number 200. The flow between three cylinders in an equilateral-triangular arrangement is investigated. The center-to-center spacing distance ratio between two circular cylinders is varied from 1.5 to 12 . Present numerical results show that the flow patterns and force characteristics are the result of the combined effects of Reynolds number, spacing distance, configuration arrangement, and incident angle. For the small spacing distance ratio of 1.5, the well-known biased flow phenomenon in the gap of downstream cylinders is found. And the biased flow is bistable in our study but not monostable. A small spacing distance means lower Strouhal number, drag, and root-mean-square lift coefficients. In the medium spacing distance ratio of 4.0, the suppressed effect of vortex shedding for the presence of the side-by-side downstream cylinders disappeared. Mean drag coefficients of downstream cylinders are basically identical to the value of flow past around a single circular cylinder. For the large spacing distance ratio of 8.0, the effects between three cylinders basically disappeared. The mean drag and lift coefficients, root-mean-square lift coefficients, and Strouhal number of three cylinders are essentially equivalent to those values of a single circular cylinder.

\section{Introduction}

Flows around circular cylinders are widespread among the modern industrial production and engineering practice, such as landing gear systems, heat exchanger tubes, offshore platforms pillar groups, and nuclear reactors. Thus, during the past century, numerous experiments and numerical simulations have been carried out to study the flow regime of flows around circular cylinders with different arrangement. Nevertheless, many works are focused on the single cylinder and a pair of cylinders [1-3]; few take consideration of three or more cylinders arrangement configuration [4]. But flow between the three cylinders is frequently involved in industrial applications; such examples are shown in Figures 1 and 2 .

Yan et al. [5], Yang et al. [6] investigated three cylinders in a staggered arrangement at $\mathrm{Re}=200$ with laser-induced fluorescence flow visualization and lattice Boltzmann numerical method. Two diverse flow characteristic steady $(1 \leq$
$T / D \leq 1.2$ and $2.5 \leq T / D \leq 3.1)$ and unsteady flow $(1.3 \leq T / D \leq 2.4$ and $3.2 \leq T / D \leq 10)$ were found. Zheng et al. [7] investigated three cylinders in an equilateral-triangular $\mathrm{T}$ shaped and inverted-T shaped configuration with the finite volume method. Three flow pattern characteristics and force characteristics were analyzed in detail. Barros et al. [8] presented the convective laminar flow and heat transfer of three cylinders in a triangular arrangement at $\mathrm{Re}=100$, Prandtl number $\operatorname{Pr}=0.71$ using finite volume method. Shaaban and Mohany [9] numerically investigated the effect of distance between the upstream cylinder and middle cylinder on the force characteristic with three uneven spacing cylinders configuration at $\mathrm{Re}=200$. Wu [10] using the secondorder immersed boundary method carried out the numerical simulation of three equilateral-triangular array cylinders with different incident angle. Bansal and Yarusevych [11] experimentally investigated flow around a cluster of three equally spaced cylinders at $\mathrm{Re}=2.1 \times 10^{3}$. POD analysis suggested that cluster orientation is a typical impact factor 


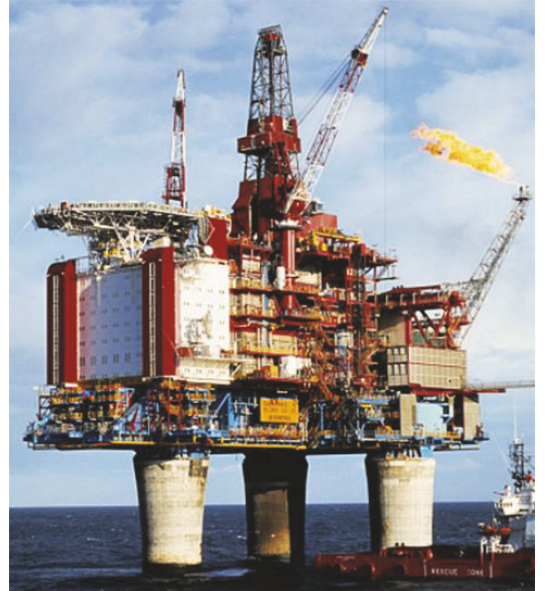

FIGURE 1: Offshore platforms pillar groups.

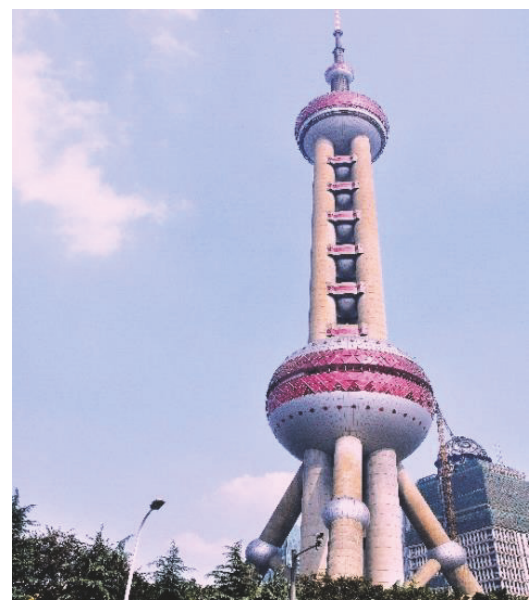

FIGURE 2: Oriental pearl radio and TV tower, Shanghai.

on the near wake development. Qiu et al. [12] experimentally measured the pressure coefficients of three cylinders in an equilateral-triangular arrangement. Significant complicated aerodynamic interference effects were observed between three cylinders. Several other literatures related to three or more cylinders are also published [13-19]. To conclude, all the above researches showed that the flow patterns and force characteristics are the results of the combined effect of Re, spacing distance, configuration arrangement, and incident angle.

With the development of hardware technology, numerical solutions can be easily obtained compared to experiment results. Numerous numerical algorithms have been used to solve the flow around cylinders, such as finite volume method, finite element method, immersed boundary method, spectral method, and meshless method. Each algorithm has pros and cons. However, in order to capture more flow details, it has been a trend to apply high resolution method into the solving of incompressible N-S equations. Spectral element method (SEM) mixes the exponential convergence property of spectral schemes and good geometric flexibility feature of finite element method. Therefore, exponential (p-type) and algebraic (h-type) convergence can be, respectively, achieved by increasing polynomial order and element numbers. In addition, the spectral element method has been applied to many research fields, such as flow and heat transfer [20,21], wave propagation [22], structure vibration [23], and aeroacoustics [24].

The paper is organized as follows. In Section 2, a brief description of the governing equation is given. In Section 3, the accuracy of the spectral element numerical code is verified by the Kovasznay flow and flow past around a single circular cylinder. Then flows between three cylinders in an equilateral-triangular arrangement are investigated in detail. Finally, Section 5 contains the conclusions of our work.

\section{Governing Equations and Numerical Methods}

The governing equations used for unsteady and incompressible viscous flow are incompressible Navier-Stokes equation and continuity equation, which are expressed as below:

$$
\begin{aligned}
\frac{\partial \mathbf{v}}{\partial t}+\mathbf{v} \cdot \nabla \mathbf{v} & =-\nabla p+\nu \nabla^{2} \mathbf{v} \\
\nabla \cdot \mathbf{v} & =0
\end{aligned}
$$

where $\mathbf{v}$ is the velocity vector, $p$ represents the pressure, $v$ is the kinematic viscosity, $v=1 /$ Re. Re is Reynold's number with respect to the circular cylinder diameter $D$ and characteristic velocity $U, \operatorname{Re}=U \cdot D / \nu$.

The numerical calculation was carried out using the open-source code Nektar++ [25]. The incompressible N$S$ equation solver in Nektar++ library is based on the spectral element method in space discretization and highorder time splitting method in time discretization. In the spectral element method, the computational domain $\Omega$ is decomposed into several nonoverlapping subdomains. Each spectral element is mapped into a standard element. Finally, the variational problem discretized by the means of SEM is equivalent to solving Differential Algebraic Equations. And the block diagram of the spectral element method for incompressible flow problems is shown in Figure 3. More details can be obtained from the literatures [20, 24, 25].

\section{Validation Study}

3.1. Kovasznay Flow. In order to validate the exponential convergence rate of the numerical scheme, we consider the two-dimensional Kovasznay flow in a rectangular area $\Omega=$ $[-0.5,1] \times[-0.5,1.5]$. This flow problem is a good test for the algorithm because an analytical closed form solution exists for the incompressible flow field. At the same time, the flow pattern is similar to the wake flow of a circular cylinder. So Kovasznay flow can be used to judge the convergence and numerical accuracy of the method for solving incompressible N-S equations. The analytical solution [29] is given by

$$
u=1-e^{\lambda x} \cos 2 \pi y
$$




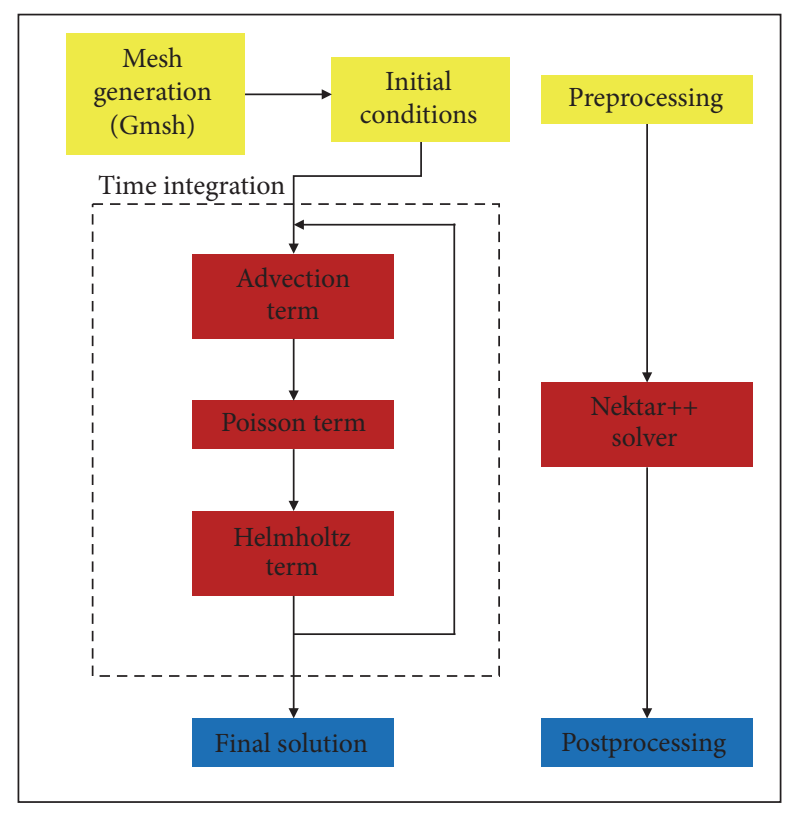

Figure 3: The block diagram of the incompressible Navier-Stokes solution algorithm.

$$
\begin{aligned}
& v=\frac{\lambda}{2 \pi} e^{\lambda x} \sin 2 \pi y \\
& p=p_{0}-\frac{1}{2} e^{2 \lambda x} \\
& \lambda=\frac{\operatorname{Re}}{2}-\sqrt{\frac{\operatorname{Re}^{2}}{4}+4 \pi^{2},}
\end{aligned}
$$

where $p_{0}$ is a reference pressure and $\lambda$ is an intermediate variable related to Re. The computational domain $\Omega$ is decomposed into 12 nonoverlapping subdomains, with 3 elements in the $\mathbf{x}$ direction and 4 elements in the $\mathbf{y}$ direction. The time step chosen is very small, so that the numerical accuracy is only connected with polynomial order. Figure 4 shows the contours of velocity component in the $x$ direction at $\mathrm{Re}=$ 40. Figure 5 shows the convergence rate of pressure and $u$ velocity. We can find that $L_{2}$ norm error has an exponential convergence rate with increasing polynomial order. The error level approaches the value of $10^{-12}$ at $P=14$. However, it does not decrease again at the higher polynomial order, which means that the calculations are reached machine error levels. Therefore, the exponential convergence of numerical scheme for solving incompressible N-S equations was verified.

3.2. Unsteady Flow Past a Single Circular Cylinder. Flow around a circular cylinder is another benchmark problem to test the accuracy of the SEM. We consider the twodimensional laminar flow past around a circular cylinder. The schematic of the numerical computation is presented in Figure 6. The center of the circular cylinder is located at the origin of the coordinate plane. The diameter of the circular cylinder $(D)$ denotes unit characteristic length. The

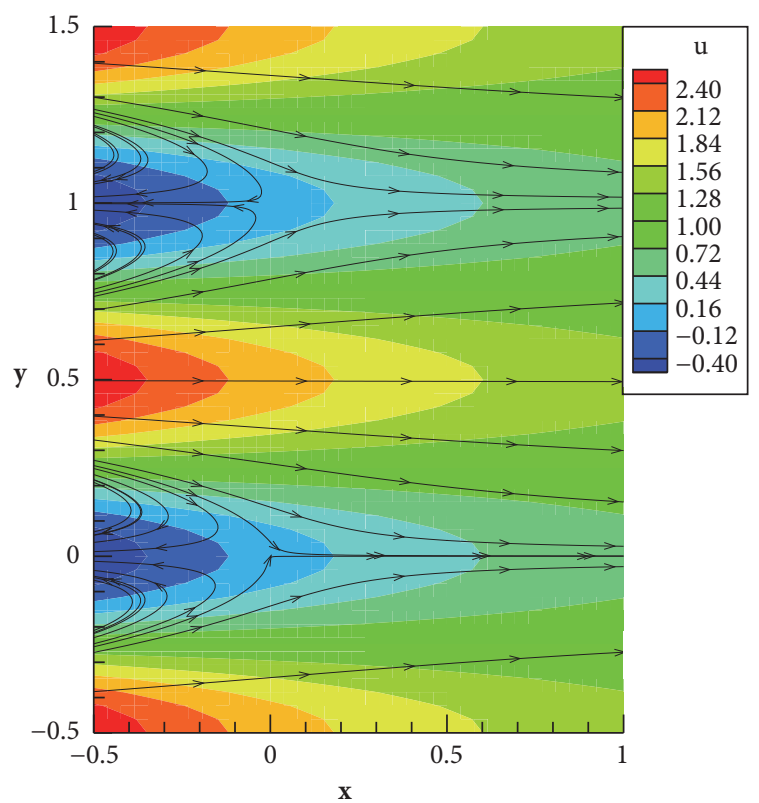

FIgURE 4: Contours of velocity component in the $x$ direction and streamlines at $\operatorname{Re}=40$.

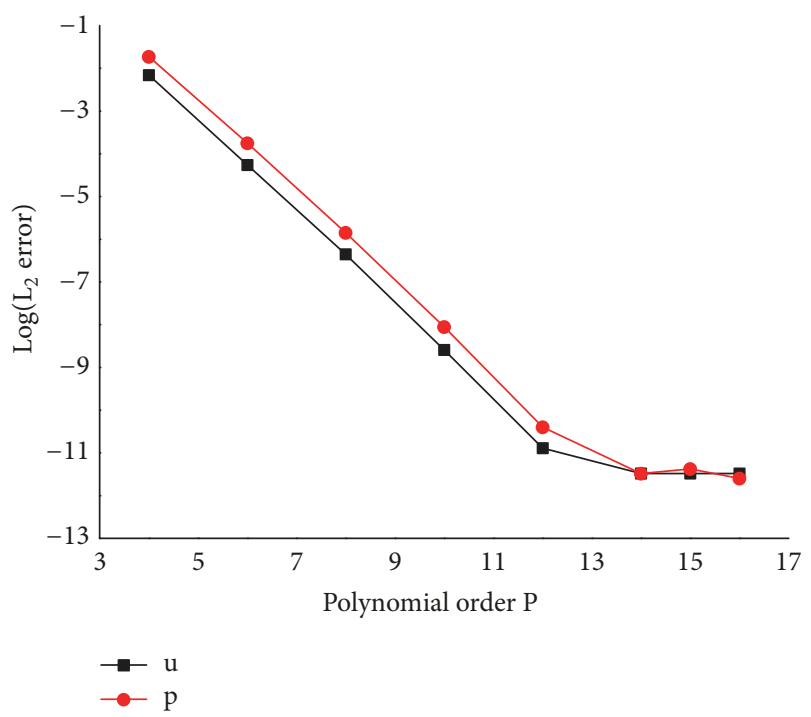

FIGURE 5: $L_{2}$ norm error convergence curves of pressure and $u$ velocity for different polynomial order at $\operatorname{Re}=40$.

entire computational domain dimensions are $50 D \times 40 D$, with a downstream distance of $30 D$, a distance of $20 D$ on both upstream, and either side of the cylinder. The boundary conditions adopted are as follows: at the inflow boundary, the velocity inlet is set to be a characteristic velocity $U=1$; at the outer boundary, zero velocity gradient and pressure $p=0$ are specified; the upper and lower boundaries are both symmetry conditions; and no-slip boundary condition is applied on the wall of circular cylinder. We run numerical computations with 1603 unstructured triangle elements and 140 structured square elements near the wall of the circular cylinder, as shown in Figures 7 and 8. In order to capture more 


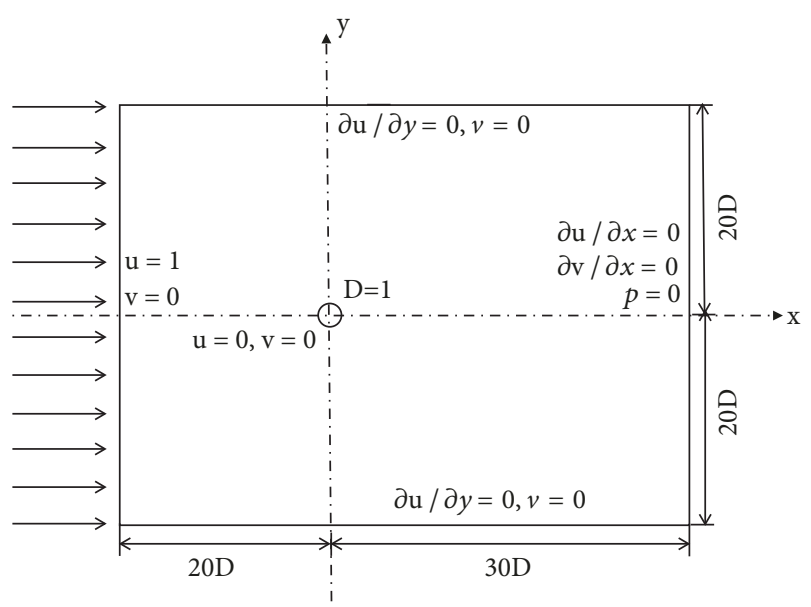

FIGURE 6: The computation domain and boundary conditions of the flow around a circular cylinder.

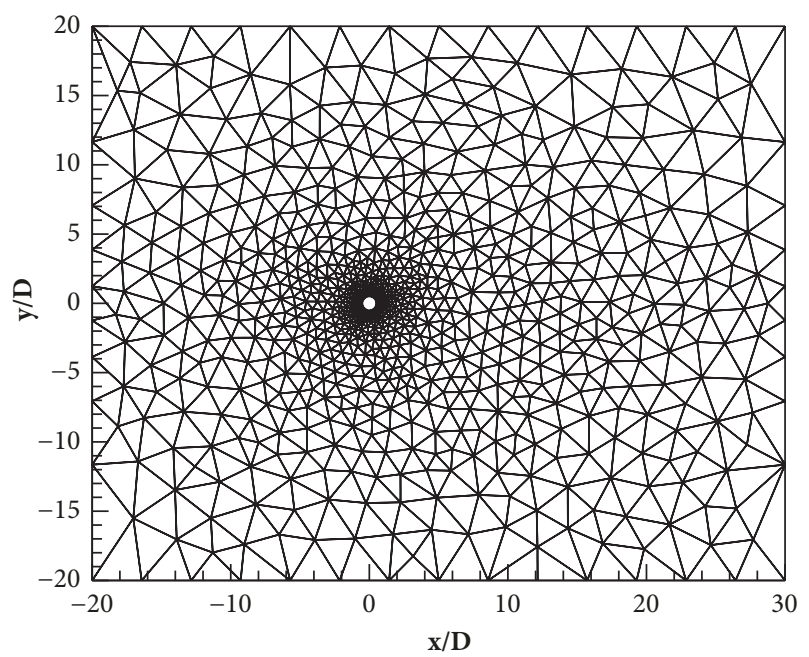

FIGURE 7: Computational grid for flow around a circular cylinder.

details of the flow near the cylinder surface, the mesh grids are imposed as narrow as $0.04 \mathrm{D}$. At the same time, the wake region is also refined. The grid systems in the present paper are all generated by Gmsh [30], open-source finite element grid software. The numerical simulation is started with zero initial conditions at $t=0$.

In this case, we fixed the grid size and a relatively small time step $\Delta t=0.0008$, by varying the polynomial order in each element; a grid independence study is investigated. The comparison of present numerical results with different polynomial orders and other existing results are given in Table 1. It is shown that using a polynomial order $P=5$, time-mean drag coefficient $\left(\overline{C_{d}}\right)$, and Strouhal number (St) provides as good a result as those obtained from previous literatures.

Some nondimensional parameters used in the present paper are defined as follows:

$$
C_{d}=\frac{2 F_{d}}{\rho U^{2} D}
$$

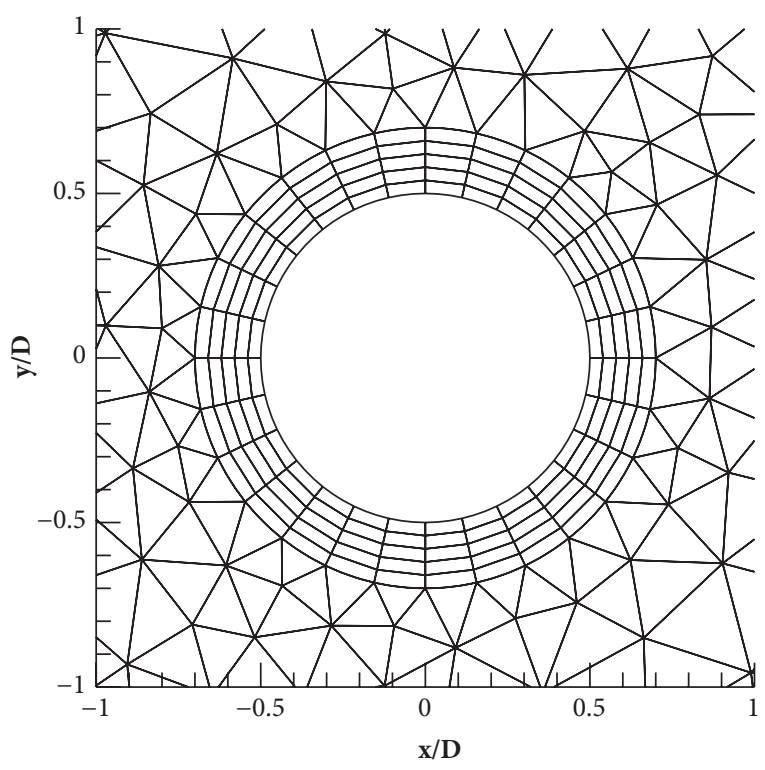

Figure 8: Local refined mesh around the circular cylinder.

TABLE 1: Comparison of the mean drag coefficients and Strouhal number.

\begin{tabular}{lcc}
\hline$P$ & $\overline{C_{d}}$ & St \\
\hline 3 & 1.3473 & 0.1999 \\
4 & 1.3463 & 0.1999 \\
5 & 1.3465 & 0.1964 \\
6 & 1.3463 & 0.1964 \\
Han et al. [26] & 1.346 & 0.1953 \\
Ding et al. [27] & 1.327 & 0.196 \\
Cai et al. [28] & 1.345 & 0.200 \\
Lam et al. [18] & 1.32 & 0.196 \\
\hline
\end{tabular}

$$
\begin{aligned}
C_{l} & =\frac{2 F_{l}}{\rho U^{2} D}, \\
\mathrm{St} & =\frac{f_{s} D}{U}, \\
C p_{\text {ave }} & =\frac{2\left(p_{\text {ave }}-p_{\infty}\right)}{\rho U^{2}}, \\
\overline{C_{d}} & =\frac{1}{T} \int_{0}^{T} C_{d} d t, \\
\overline{C_{l}} & =\frac{1}{T} \int_{0}^{T} C_{l} d t, \\
C_{l}^{\prime} & =\sqrt{\frac{1}{T} \int_{0}^{T} C_{l}^{2} d t .}
\end{aligned}
$$

where $F_{d}, C_{d}$ are the drag force and drag coefficient; $F_{l}, C_{l}$ are the lift force and lift coefficient; $f_{s}$ is the vortex shedding frequency, which can be obtained from the FFT analysis of the lift coefficient curve; $C p_{\text {ave }}$ is mean pressure coefficient; $p_{\text {ave }}$ 


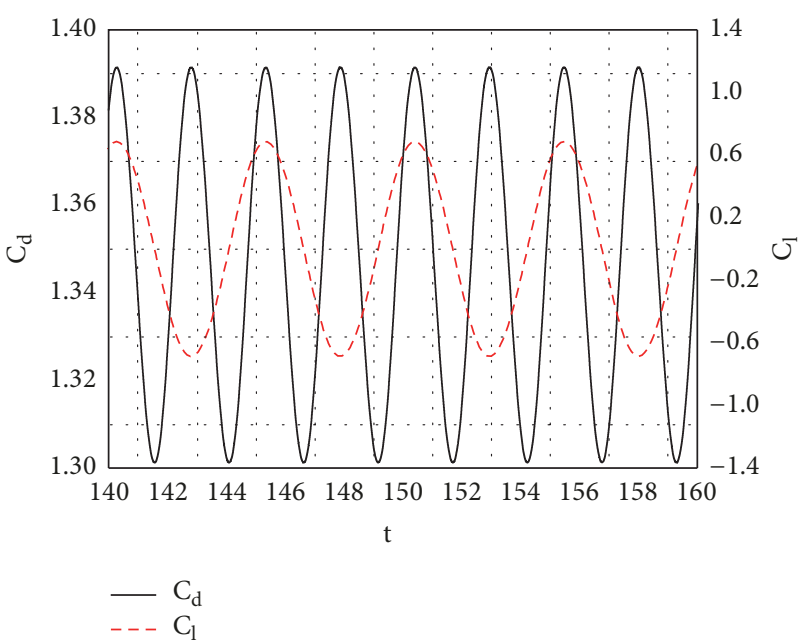

FIgURE 9: Temporal evolution curve of lift coefficients and drag coefficients.

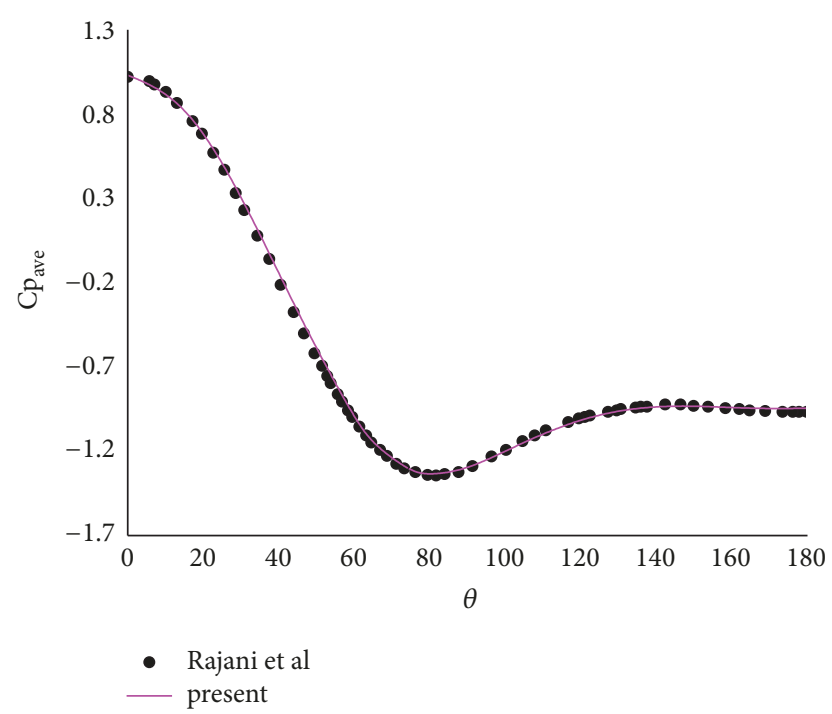

FIGURE 10: Distribution of time averaged pressure coefficients on the surface of circular cylinder.

is mean pressure; $p, p_{\infty}$ are free stream pressure; $\rho$ is fluid density; $T$ is one stable period; $\overline{C_{l}}, C_{l}^{\prime}$ are the time-mean and root-mean-square lift coefficients.

Figure 9 shows the temporal evolution curve of lift coefficients and drag coefficients at $\operatorname{Re}=200$. As what can be seen from the graph shown in Figure 9, the vortex shedding has developed to be a stable periodic fashion. The period of lift coefficient is twice the period of drag coefficient. In addition, the time averaged pressure coefficient on the surface of circular cylinder is also an important parameter for charactering the flow around a circular cylinder. Figure 10 shows the comparison of $C p_{\text {ave }}$ between our numerical result and the literature results from [31]. Good agreement is observed, and it shows the capabilities of the spectral element method encapsulated in Nektar++ for solving the incompressible flow problems again.

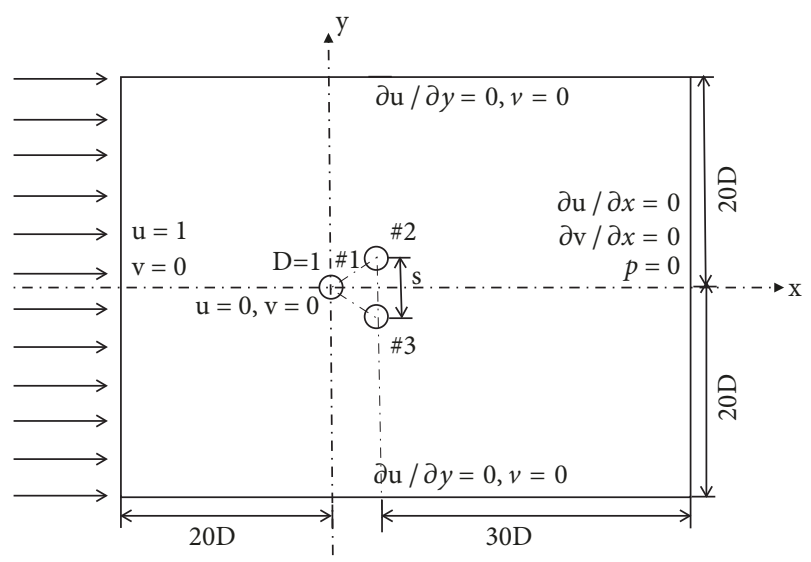

FIGURE 11: The computation domain and boundary conditions of the flow around three circular cylinders.

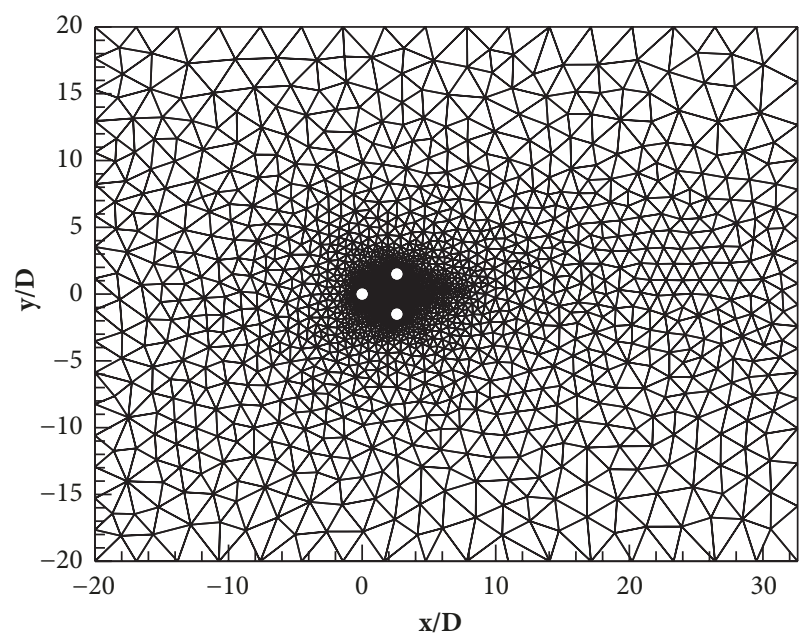

FIGURE 12: Computational grid for flow around three circular cylinders with $S / D=4.0$.

\section{Unsteady Flow between Three Circular Cylinders}

Now, we consider the two-dimensional laminar flow past between three circular cylinders. The schematic of the numerical computation is presented in Figure 11. Three circular cylinders are placed in the shape of an equilateral triangle. The center of the upstream circular cylinder (denoted with cylinder 1) is always located at the origin of the coordinates. Another two cylinders are located at downstream in a sideby-side configuration (denoted with cylinder 2 and 3, respectively). $S$ is the center-to-center spacing between two circular cylinders. The boundary conditions are the same as the ones adopted by flow past a single cylinder. Computational grid for flow around three circular cylinders with $S / D=4.0$ is presented in Figures 12 and 13. 3893 unstructured triangle elements and 330 structured square elements are used for the case $S / D=4.0$. Just like the single circular cylinder, we fix the grid size and time step, by varying the polynomial order in each element; a grid independence test is also examined. 
TABLE 2: Comparison of the mean drag coefficient and Strouhal number of flow around three cylinders.

\begin{tabular}{cccc}
\hline$P$ & $\overline{C_{d}}$ & St \\
\hline \multirow{3}{*}{3} & & 1.1814 & 0.18 \\
& $\# 1$ & 1.3470 & 0.18 \\
& $\# 3$ & 1.3465 & 0.18 \\
4 & $\# 1$ & 1.1811 & 0.18 \\
& $\# 2$ & 1.3459 & 0.18 \\
& $\# 3$ & 1.3459 & 0.18 \\
5 & $\# 1$ & 1.1810 & 0.18 \\
& $\# 2$ & 1.3459 & 0.18 \\
& $\# 3$ & 1.3460 & 0.18 \\
\hline
\end{tabular}

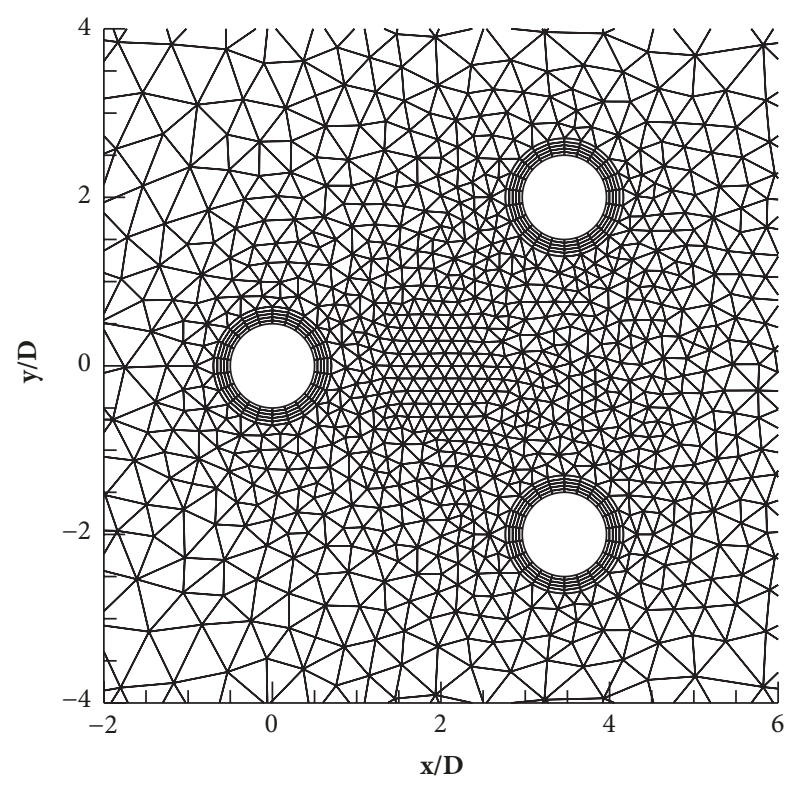

FIGURE 13: Local refined mesh around the three circular cylinders.

Table 2 displays the comparison of $\overline{C_{d}}$ and St between different polynomial orders. Owing to the enough element numbers, it is shown that grid independence is obtained using a polynomial order $P=3$. However, in order to present the flow patterns more clearly, the polynomial order $P=4$ is adopted in the below numerical simulations.

4.1. Flow Patterns. Previous research had identified that the spacing distance has a vital impact on the flow pattern characteristics. Therefore, in this paper, we do a study of the spacing distance $S / D$ from 1.5 to 12 at $\mathrm{Re}=200$. The flow pattern characteristics of three typical spacing distances are discussed in below. Figures 14-16 show presented computed results for instantaneous vorticity contours, pressure contours, and streamlines at $S / D=1.5,4.0$, and 8.0 with $\operatorname{Re}=200$.

For the small spacing distance case of $S / D=1.5$, crossflow around three cylinders with an equilateral-triangular arrangement is similar to a single bluff body flow. As can be seen from Figure 14(a), the single-row vortex street was produced in the downstream of wake region. Vortex shedding of upstream cylinder is fully suppressed due to the presence of the side-by-side downstream cylinders. The existence of wellknown biased flow phenomenon in the gap of downstream cylinders is proved. And owing to this asymmetry flow, the flow pattern becomes irregularly and spontaneously. Moreover, it is of interest that we find the biased flow is bistable in our study, but not monostable in literatures [7, 16]. This is also noticed by Sumner [1], Zhiwen et al. [32]. They explained that the bistable characteristic was related to the transient presentation and breakdown of large gap vortices behind the cylinder with the wider wake. $\mathrm{Gu}$ and Sun [33] also found this biased bistable flow at $\mathrm{Re}=14000$ with $S / D=1.7$. One reason for the inconsistency between different researchers may be linked to the mixed effects of changes in Re and spacing distance. Further comprehensive research for this biased flow phenomenon should be carried out.

For the medium spacing distance case of $S / D=4.0$, Figures 15(a) and 15(b) showed that cross-flow around three cylinders with an equilateral-triangular arrangement are all partially developed. The three-row vortex street was produced in the downstream of wake region. The suppressed effect of vortex shedding for the presence of the side-by-side downstream cylinders is disappeared. Gap flow is no longer dominated by the downstream cylinders. And every cylinder produces an obvious single vortex street in the wake region. The wake of upstream cylinder is sandwiched by the wakes of the side-by-side downstream cylinders. Owing to this restriction, the wake extent of upstream cylinder becomes more relatively narrow, while those at the downstream become wider. Moreover, the vortex shedding behind three cylinders nearly presents an in-phase synchronized fashion. The results show that there is a strong interaction between the vortex shedding of cylinder $\# 1$ with those from the inner sides of downstream cylinders \#2 and \#3. However, the vortex shedding of the outer sides of downstream cylinders \#2 and \#3 does not take part in this merging process.

For the large spacing distance case of $S / D=8.0$, as shown in Figures 16(a) and 16(b), cross-flow around three cylinders with an equilateral-triangular arrangement is all completely developed. The three-row well-defined vortex street was produced in the downstream of wake region. The flow pattern of any one of three cylinders only has a slight difference with that of cross-flow around a single cylinder. And this slight difference can be seen from the instantaneous streamlines in the wake region of Figure 16(b). Vortex shedding between cylinder \#1 and cylinder \#2 nearly presents an in-phase synchronized fashion. On the contrary, Vortex shedding between cylinder \#2 and cylinder \#3 nearly presents an antiphase synchronized fashion.

4.2. Force Statistics. Figures 17-19 show the temporal evolution curve of lift and drag coefficients for three cylinders at $S / D=1.5,4.0$, and 8.0. Variations of mean drag coefficients, mean lift coefficients, root-mean-square lift coefficients, and St with different spacing distances for three cylinders are illustrated in Figures 20-23.

Due to the existence of the biased flow phenomenon in the case of $S / D=1.5$, the first impression for curves in Figure 17 is of very large irregular and disordered. The 


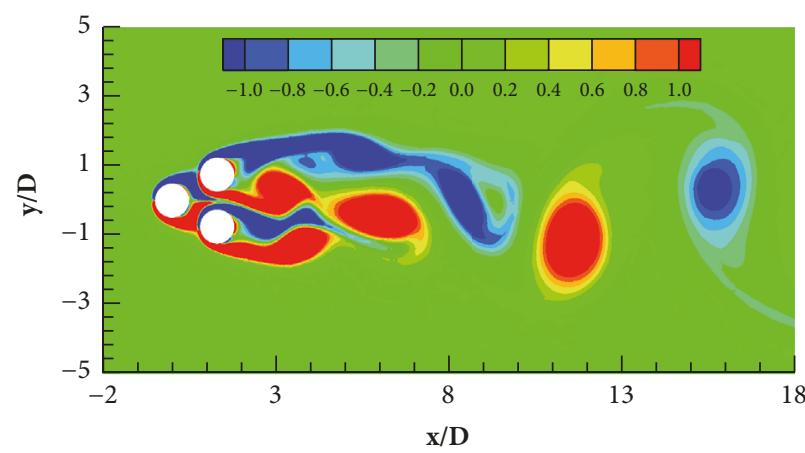

(a)

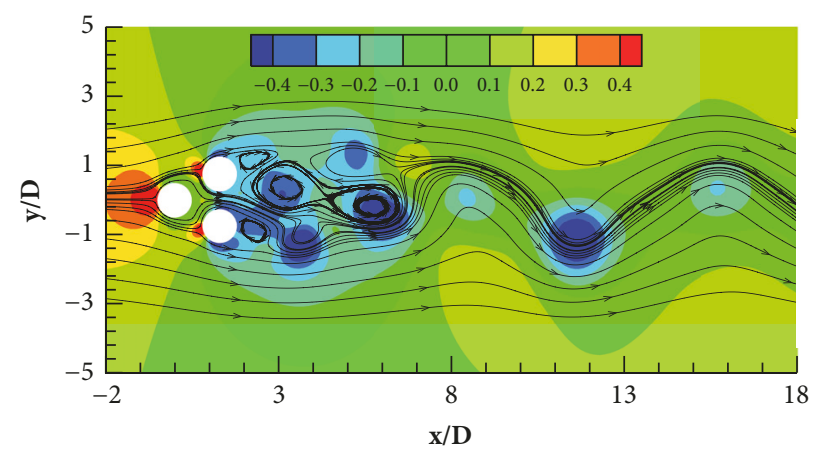

(b)

Figure 14: Snapshots of instantaneous flow fields at $S / D=1.5$ with $\mathrm{Re}=200$ : (a) vorticity contours and (b) pressure contours and streamlines.

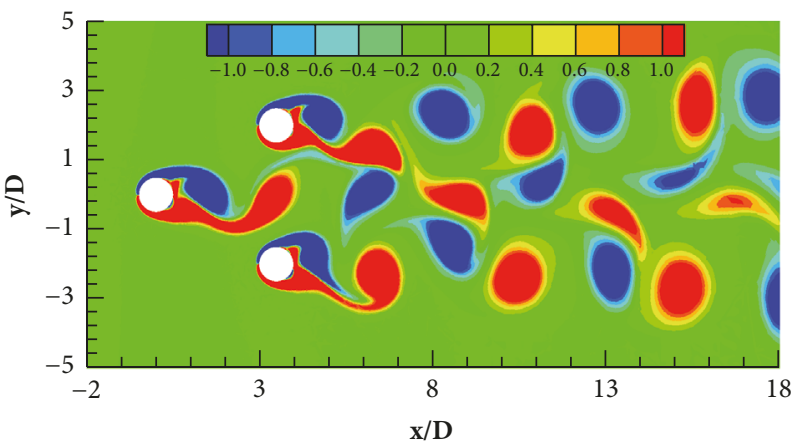

(a)

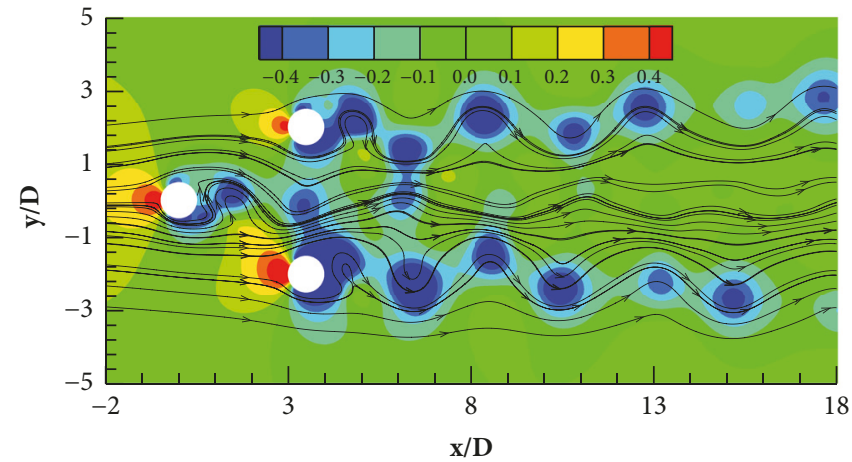

(b)

FIGURE 15: Snapshots of instantaneous flow fields at $S / D=4.0$ with $\operatorname{Re}=200$ : (a) vorticity contours and (b) pressure contours and streamlines.

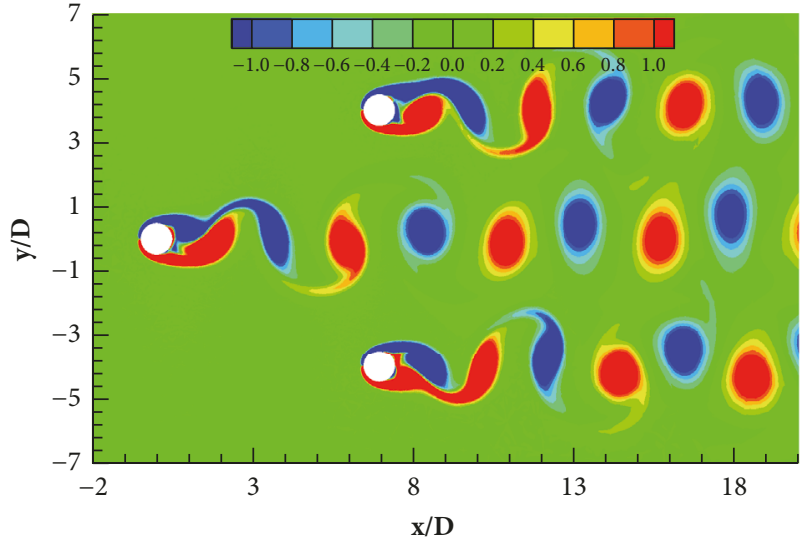

(a)

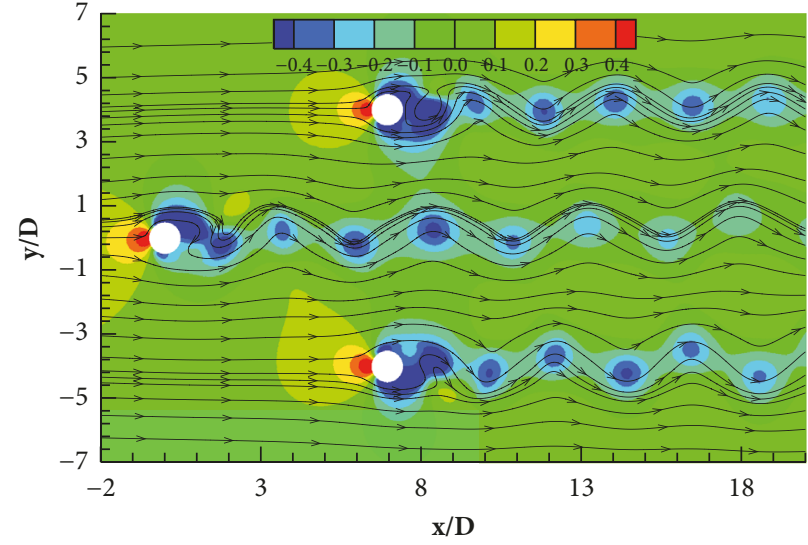

(b)

FIGURE 16: Snapshots of instantaneous flow fields at $S / D=8.0$ with $\mathrm{Re}=200$ : (a) vorticity contours and (b) pressure contours and streamlines.

drag and lift coefficients of cylinder \#1 are all obviously less than the values of other two cylinders. The mean drag and RMS lift coefficients of cylinders \#2 and \#3 are basically identical. Lift coefficients have the nearly the same magnitude but opposite sign. The St of three circular cylinders is small enough in comparison with the value of flow past around a single circular cylinder. Therefore, it can be concluded that a small spacing distance tends to have a lower St, drag, and RMS lift coefficients.
In the medium spacing distance case of $S / D=4.0$, all coefficients versus time history present a sinusoidal shape except the drag coefficients of cylinders 2\# and 3\#. Drag coefficients of cylinders \#2 and \#3 have the same magnitude, but some difference in phase. The phase of lift coefficients for three cylinders is all identical, and this also suggests the same St will be obtained. This character can be attributed to the inphase synchronized vortex shedding behind three cylinders. The mean drag coefficients of cylinders $\# 2$ and $\# 3$ are basically 


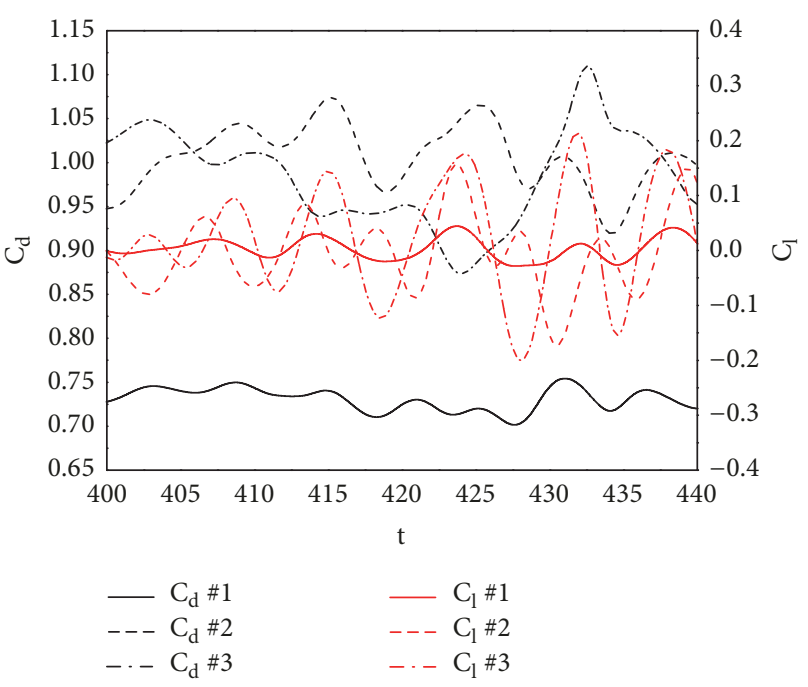

FIGURE 17: Temporal evolution curve of lift and drag coefficients for three cylinders at $S / D=1.5$.

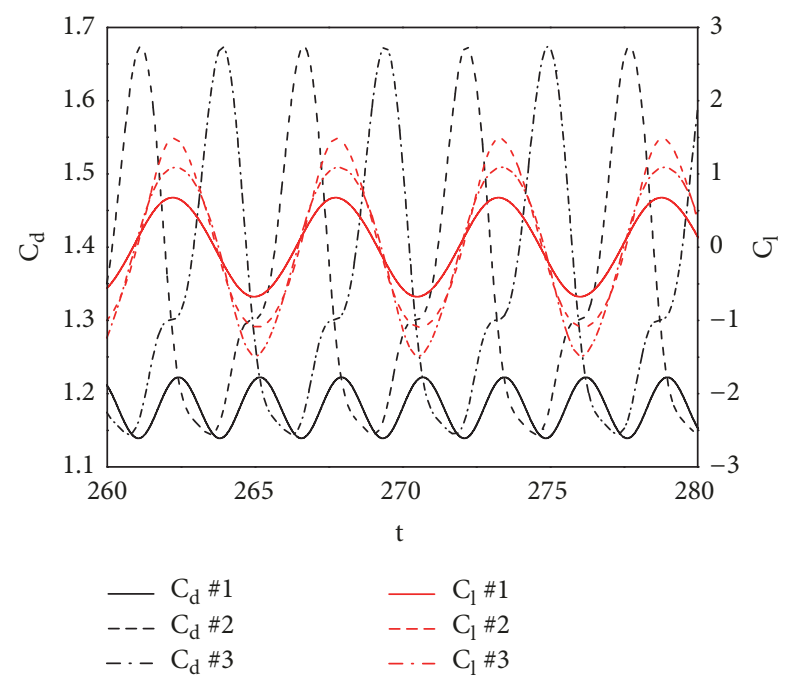

Figure 18: Temporal evolution curve of lift and drag coefficients for three cylinders at $S / D=4.0$.

identical to the value of flow past around a single circular cylinder, except for the fact that the mean drag coefficients of cylinder \#1 are a bit less than the value of a single circular cylinder. The reason for this can be referred to the strong interaction between the vortex shedding of cylinder \#1 with those from the inner sides of downstream cylinders \#2 and \#3.

For the large spacing distance case of $S / D=8.0$, due to the antiphase synchronized vortex shedding, temporal evolution curves of drag coefficients for cylinders \#2 and \#3 are in-phase. Moreover, the maximum drag coefficients of cylinder \#2 correspond to the minimum drag coefficients of cylinder \#3, and vice versa. The mean drag coefficients of three cylinders are basically identical to the value of the single circular cylinder. The cylinder $\# 1$ is a bit less than it, and the other two cylinders are a bit bigger than it. As is shown in Figure 23, the St of three cylinders maintains the same

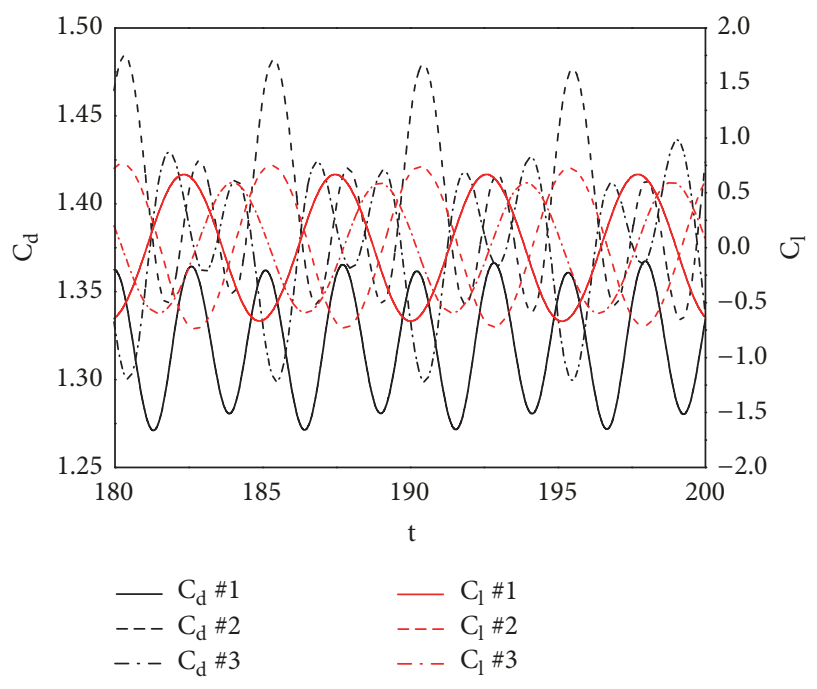

FIGURE 19: Temporal evolution curve of lift and drag coefficients for three cylinders at $S / D=8.0$.

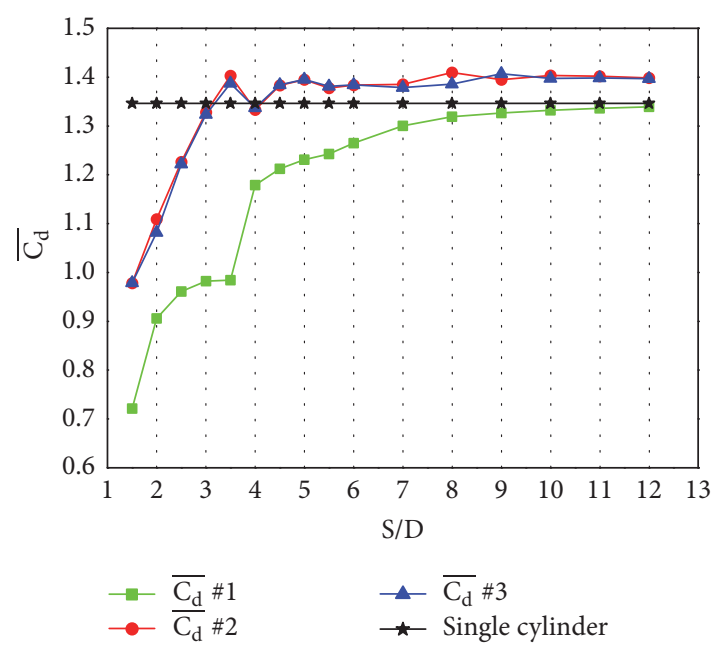

FIGURE 20: Variations of mean drag coefficients with different spacing distances for three cylinders.

value. Also, the St of three cylinders is slightly larger than the value of a single cylinder. Therefore, it can be concluded that there is also a slight difference between the flow past around a single circular cylinder and three cylinders with an equilateral-triangular arrangement in the large spacing distance case of $S / D=8.0$.

With the increase of the spacing distance, the mean drag coefficients become increasingly obvious. The growth rate of cylinder \#1 is slower than the other two cylinders, and there is an abrupt jump in the mean drag coefficients of cylinder \#1. This also can be seen from Figures 21 and 22. Keep increasing the spacing distance, the mean drag coefficients of three cylinders maintain a constant number, respectively.

Additionally, mean lift coefficients have the nearly same magnitude but opposite sign, as illustrated in Figure 21. This is caused by the negative pressure distributions around the cylinder \#2 and \#3. Mean lift coefficients of upstream cylinder 


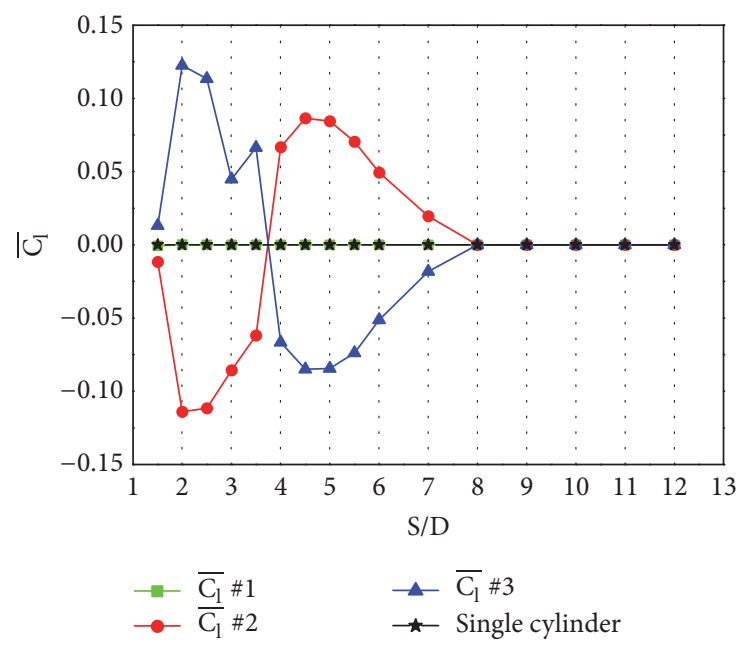

FIGURE 21: Variations of mean lift coefficients with different spacing distances for three cylinders.

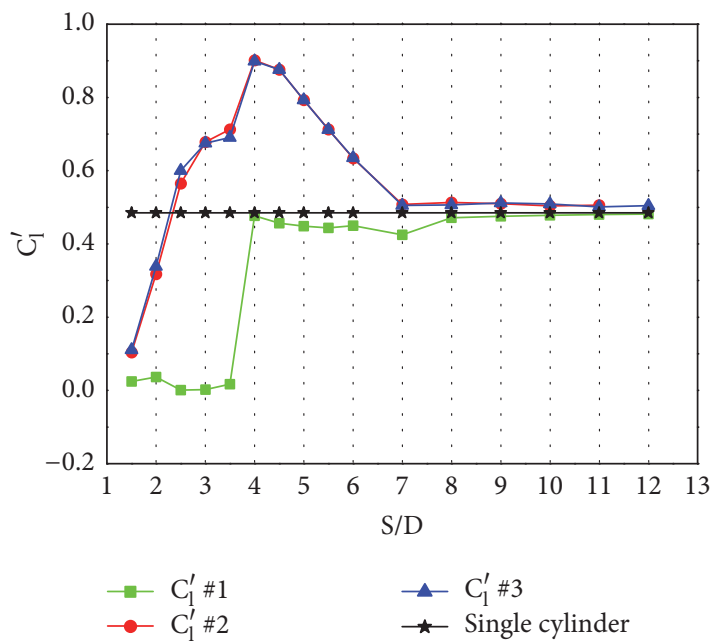

FIGURE 22: Variations of RMS lift coefficients with different spacing distances for three cylinders.

always keep a constant value of 0 , which is coinciding with the result of a single cylinder. RMS lift coefficients of cylinders $\# 2$ and \#3 reach a maximum at $S / D=4.0$ and then decline gradually to 0 at $S / D=8.0$ and thereafter maintain this value with the bigger spacing distance. RMS lift coefficients of cylinders \#1 reach a maximum at $S / D=4.0$ and then maintain this value, which is close to that obtained by a single cylinder. The St of three cylinders become increasingly obvious with small spacing distance. In the medium spacing distance, the St are all less than the value of a single cylinder. However, in the large spacing distance, the St are all greater than the value of a single cylinder. We also found that the St of three cylinders are almost equivalent to each other for all spacing distances.

\section{Conclusions}

In this paper, the spectral element method which combines the advantages of the spectral method and finite element

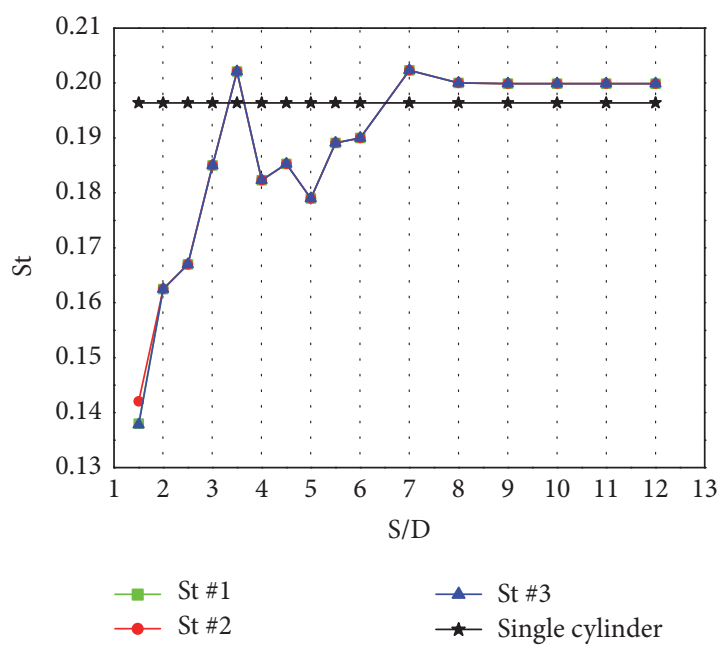

FIGURE 23: Variations of St with different spacing distances for three cylinders.

method was adopted to solve the flow between three cylinders in an equilateral-triangular arrangement. The simulation results of Kovasznay flow and flow past around a single circular cylinder showed a good consistency with the available literatures. And this indicated that the algorithm was efficient and high-order accuracy.

For the small spacing distance case of $S / D=1.5$, the existence of well-known biased flow phenomenon in the gap of downstream cylinders was proved. However, it is of interest that we found the biased flow is bistable in our study but not monostable. A small spacing distance presented lower St, drag, and RMS lift coefficients.

In the medium spacing distance case of $S / D=4.0$, the suppressed effect of vortex shedding for the presence of the side-by-side downstream cylinders disappeared. The results showed that there was a strong interaction between the vortex shedding of cylinder \#1 with those from the inner sides of downstream cylinders \#2 and \#3. The mean drag coefficients of cylinders \#2 and \#3 were basically identical to the value of flow past around a single circular cylinder, except for the fact that the mean drag coefficients of cylinder \#1 were a bit less than the value of a single circular cylinder.

For the large spacing distance case of $S / D=8.0$, the flow pattern of any one of three cylinders only has a slight difference with that of cross-flow around a single cylinder. The mean drag and lift coefficients, RMS lift coefficients, and St of three cylinders were essentially equal to the value of a single circular cylinder. This result illustrated that the effects between three cylinders basically disappeared for $S / D \geq 8.0$.

\section{Data Availability}

The data used to support the findings of this study are available from the corresponding author upon request.

\section{Conflicts of Interest}

The authors declare that they have no conflicts of interest. 


\section{Acknowledgments}

This work was supported by the National Natural Science Foundation of China (Grant no. 51776155).

\section{References}

[1] D. Sumner, "Two circular cylinders in cross-flow: a review," Journal of Fluids and Structures, vol. 26, no. 6, pp. 849-899, 2010.

[2] Y. Zhou and M. M. Alam, "Wake of two interacting circular cylinders: a review," International Journal of Heat and Fluid Flow, vol. 62, pp. 510-537, 2016.

[3] Z. Li, M. A. Prsic, M. C. Ong, and B. C. Khoo, "Large Eddy Simulations of flow around two circular cylinders in tandem in the vicinity of a plane wall at small gap ratios," Journal of Fluids and Structures, vol. 76, pp. 251-271, 2018.

[4] L. Ma, Y. Gao, Z. Guo, and L. Wang, "Experimental investigation on flow past nine cylinders in a square configuration," Fluid Dynamics Research, vol. 50, no. 2, pp. 1-47, 2018.

[5] W. Yan, J. Wu, S. Yang, and Y. Wang, "Numerical investigation on characteristic flow regions for three staggered stationary circular cylinders," European Journal of Mechanics-B/Fluids, vol. 60, pp. 48-61, 2016.

[6] S. Yang, W. Yan, J. Wu, C. Tu, and D. Luo, "Numerical investigation of vortex suppression regions for three staggered circular cylinders," European Journal of Mechanics-B/Fluids, vol. 55, no. part 1, pp. 207-214, 2016.

[7] S. Zheng, W. Zhang, and X. Lv, "Numerical simulation of crossflow around three equal diameter cylinders in an equilateraltriangular configuration at low Reynolds numbers," Computers \& Fluids, vol. 130, pp. 94-108, 2016.

[8] G. M. Barros, G. Lorenzini, L. A. Isoldi, L. A. O. Rocha, and E. D. dos Santos, "Influence of mixed convection laminar flows on the geometrical evaluation of a triangular arrangement of circular cylinders," International Journal of Heat and Mass Transfer, vol. 114, pp. 1188-1200, 2017.

[9] M. Shaaban and A. Mohany, "Flow-induced vibration of three unevenly spaced in-line cylinders in cross-flow," Journal of Fluids and Structures, vol. 76, pp. 367-383, 2018.

[10] Y. L. Wu, "Numerical simulation of flows past multiple cylinders using the hybrid local domain free discretization and immersed boundary method," Ocean Engineering, vol. 141, pp. 477-492, 2017.

[11] M. S. Bansal and S. Yarusevych, "Experimental study of flow through a cluster of three equally spaced cylinders," Experimental Thermal and Fluid Science, vol. 80, pp. 203-217, 2017.

[12] X. Qiu, H. Liu, M. He, R. He, and J. Dong, "Experimental study for the cross-flow around three cylinders in an isosceles right triangle configuration," Journal of Wind Engineering \& Industrial Aerodynamics, vol. 170, pp. 185-196, 2017.

[13] Y. Bao, Q. Wu, and D. Zhou, "Numerical investigation of flow around an inline square cylinder array with different spacing ratios," Computers \& Fluids, vol. 55, pp. 118-131, 2012.

[14] A. B. Harichandan and A. Roy, "Numerical investigation of low Reynolds number flow past two and three circular cylinders using unstructured grid CFR scheme," International Journal of Heat and Fluid Flow, vol. 31, no. 2, pp. 154-171, 2010.

[15] Z. Han, D. Zhou, T. He et al., "Flow-induced vibrations of four circular cylinders with square arrangement at low Reynolds numbers," Ocean Engineering, vol. 96, pp. 21-33, 2015.
[16] M. Tatsuno, H. Amamoto, and K. Ishi-i, "Effects of interference among three equidistantly arranged cylinders in a uniform flow," Fluid Dynamics Research, vol. 22, no. 5, pp. 297-315, 1998.

[17] M. Zhao, K. Kaja, Y. Xiang, and L. Cheng, "Vortex-induced vibration of four cylinders in an in-line square configuration," Physics of Fluids, vol. 28, no. 2, pp. 1-31, 2016.

[18] K. Lam, W. Q. Gong, and R. M. C. So, "Numerical simulation of cross-flow around four cylinders in an in-line square configuration," Journal of Fluids and Structures, vol. 24, no. 1, pp. 34-57, 2008.

[19] E. Feldshtein, J. Józwik, and S. Legutko, "The influence of the conditions of emulsion mist formation on the surface roughness of aisi 1045 steel after finish turning," Advances in Science and Technology Research Journal, vol. 10, no. 30, pp. 144-149, 2016.

[20] G. Tang, L. Cheng, F. Tong, L. Lu, and M. Zhao, "Modes of synchronisation in the wake of a streamwise oscillatory cylinder," Journal of Fluid Mechanics, vol. 832, pp. 146-169, 2017.

[21] D. Liu, Y.-L. Zheng, A. Moore, and M. Ferdows, "Spectral element simulations of three dimensional convective heat transfer," International Journal of Heat and Mass Transfer, vol. 111, pp. 1023-1038, 2017.

[22] M. R. Machado and J. M. C. Dos Santos, "Reliability analysis of damaged beam spectral element with parameter uncertainties," Shock and Vibration, vol. 2015, Article ID 574846, 12 pages, 2015.

[23] Y.-X. Huang, H. Tian, and Y. Zhao, "Effects of cable on the dynamics of a cantilever beam with tip mass," Shock and Vibration, vol. 2016, Article ID 7698729, 11 pages, 2016.

[24] A. Beck and C.-D. Munz, "Direct aeroacoustic simulations based on high order discontinuous Galerkin schemes," in Computational Acoustics, vol. 579 of CISM Courses and Lect., pp. 159-204, Springer, Cham, 2018.

[25] C. D. Cantwell, D. Moxey, A. Comerford et al., "Nektar++: an open-source spectral/hp element framework," Computer Physics Communications, vol. 192, pp. 205-219, 2015.

[26] Z. Han, D. Zhou, X. Gui, and J. Tu, "Numerical study of flow past four square-arranged cylinders using spectral element method," Computers \& Fluids, vol. 84, pp. 100-112, 2013.

[27] H. Ding, C. Shu, K. S. Yeo, and D. Xu, "Simulation of incompressible viscous flows past a circular cylinder by hybrid FD scheme and meshless least square-based finite difference method," Computer Methods Applied Mechanics and Engineering, vol. 193, no. 9-11, pp. 727-744, 2004.

[28] S.-G. Cai, A. Ouahsine, J. Favier, and Y. Hoarau, "Moving immersed boundary method," International Journal for Numerical Methods in Fluids, vol. 85, no. 5, pp. 288-323, 2017.

[29] L. I. G. Kovasznay, "Laminar flow behind a two-dimensional grid," Mathematical Proceedings of the Cambridge Philosophical Society, vol. 44, no. 1, pp. 58-62, 1948.

[30] C. Geuzaine and J. F. Remacle, "Gmsh: a 3-D finite element mesh generator with built-in pre- and post-processing facilities," International Journal for Numerical Methods in Engineering, vol. 79, no. 11, pp. 1309-1331, 2009.

[31] B. N. Rajani, A. Kandasamy, and S. Majumdar, "Numerical simulation of laminar flow past a circular cylinder," Applied Mathematical Modelling: Simulation and Computation for Engineering and Environmental Systems, vol. 33, no. 3, pp. 1228-1247, 2009.

[32] W. Zhiwen, Y. Zhou, and H. Li, "Flow-visualization of a two side-by-side cylinder wake," Journal of Flow Visualization and Image Processing, vol. 9, no. 2, pp. 121-138, 2002. 
[33] Z. Gu and T. Sun, "Classification of flow pattern on three circular cylinders in equilateral-triangular arrangements," Journal of Wind Engineering \& Industrial Aerodynamics, vol. 89, no. 6, pp. 553-568, 2001. 


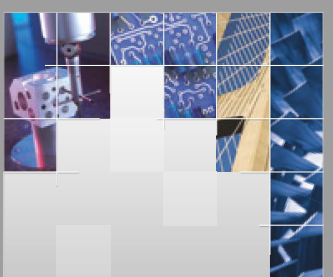

\section{Enfincering}
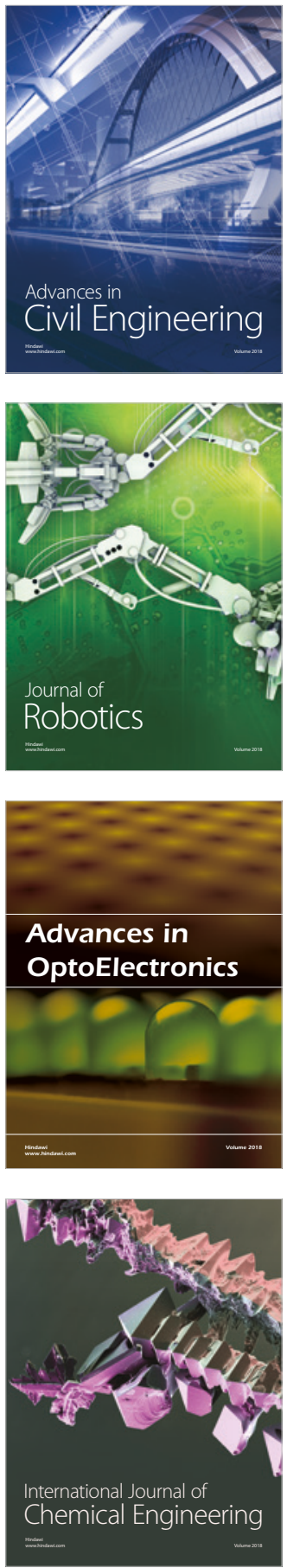

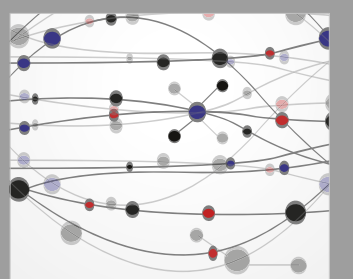

\section{Rotating \\ Machinery}

The Scientific World Journal

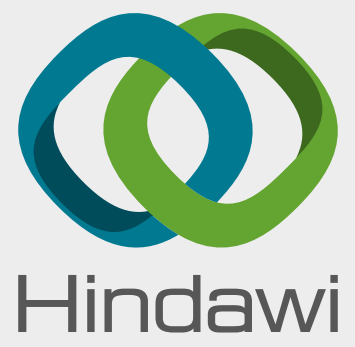

Submit your manuscripts at

www.hindawi.com
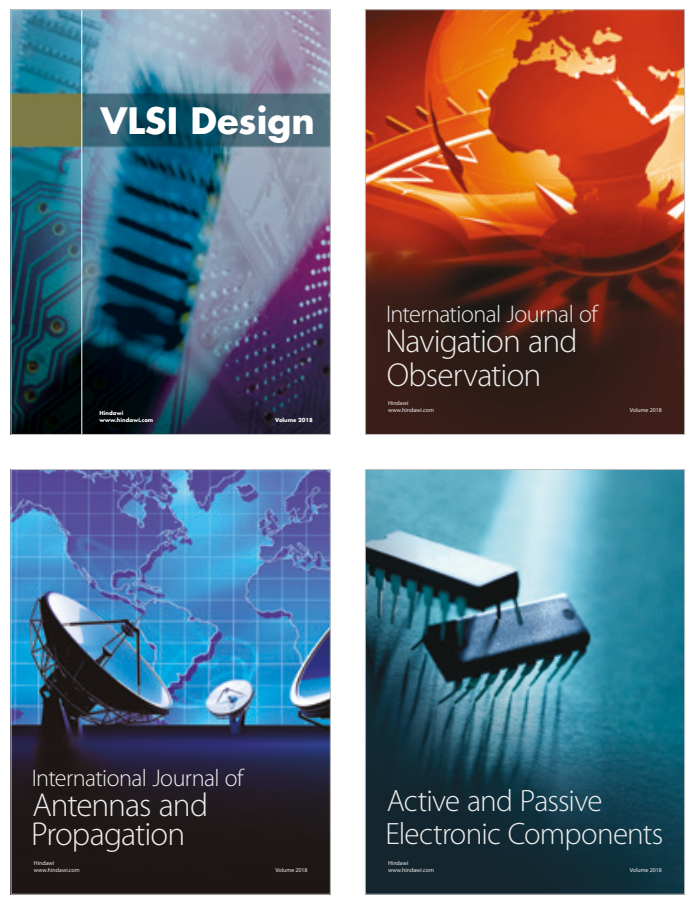
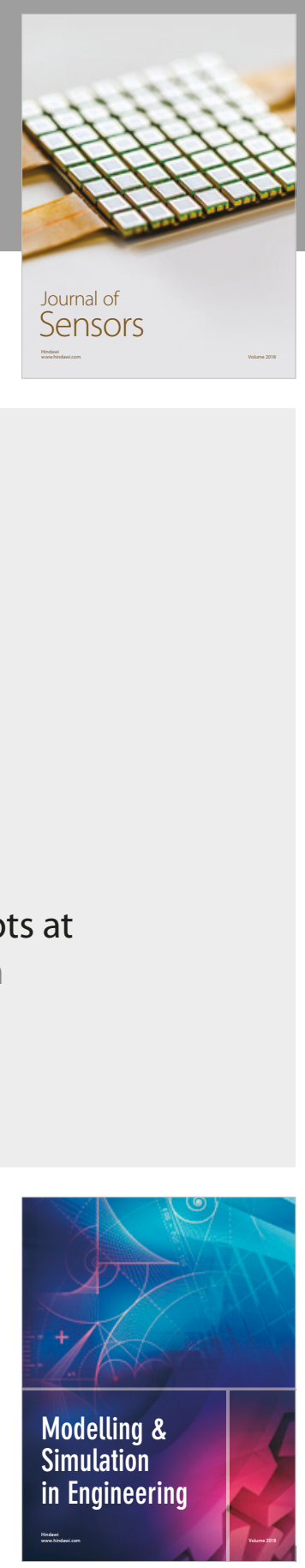

\section{Advances \\ Multimedia}
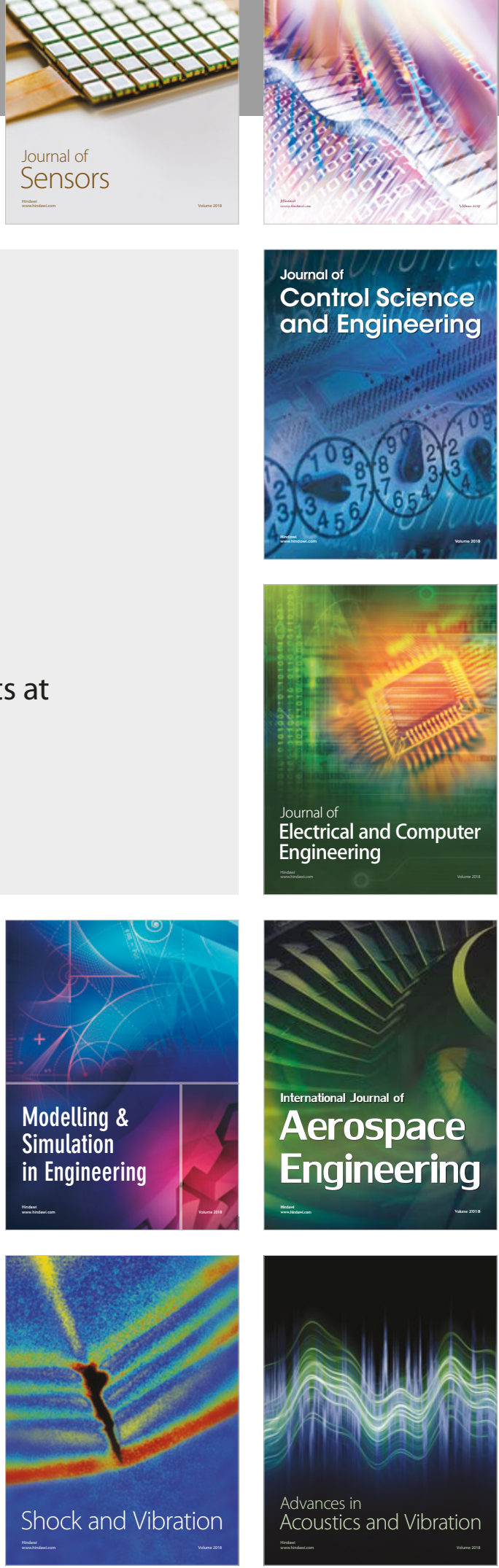